\title{
Computation of elastic moduli of nanocrystalline materials using Voronoi models of representative volume elements
}

\author{
Panagiotis Bazios ${ }^{1}$, Konstantinos Tserpes ${ }^{1, *}$, and Spiros Pantelakis ${ }^{1}$ \\ ${ }^{1}$ Laboratory of Technology and Strength of Materials, Department of Mechanical Engineering and \\ Aeronautics, University of Patras, 26500 Rion, Greece
}

\begin{abstract}
In the present work, a numerical model is developed to predict the Young's modulus and shear modulus of nanocrystalline materials using a Finite Element Analysis. The model is based on Representative Volume Elements (RVE) in which the microstructure of the material is described using the Voronoi tessellation algorithm. The use of the Voronoi particles was based on the observation of the morphology of nanocrystalline materials by Scanning Electron and Transmission Electron Microscopy. In each RVE, three-dimensional modelling of the grain and grain boundaries as randomlyshaped sub-volumes is performed. The developed model has been applied to pure nanocrystalline copper at grain volume fractions of $80 \%, 90 \%$ and $95 \%$ taking also into account the parameters of grain size and grain boundary thickness. The elastic moduli of nanocrystalline copper have been computed by loading the RVE in tension. The numerical results reveal that the elastic moduli of nanocrystalline copper increase with increasing the grain volume fraction. On the other hand, for a given grain volume fraction, the results showed no effect of the grain size. The model predictions have been validated successfully against numerical results from the literature and predictions of the Rule of Mixtures and the Mori-Tanaka analytical model.
\end{abstract}

\section{Introduction}

Nanocrystalline (NC) metals have attracted considerable interest over the past two decades due to their unique mechanical properties [1]. One of the features of these materials, which is best examined and described, is their mechanical behavior [2]. It is commonly known that compared with their microcrystalline counterparts, nanomaterials are characterized by higher values of the yield stress, tensile strength, and hardness, whereas their plasticity and fracture toughness are lower [3]. Another basic parameters which describe the mechanical behavior of materials are their modulus of elasticity - E and Shear Modulus - G. In the case of nanomaterials there are however some problems with determining the elastic moduli. The possible relation between the value of this modulus and the structure of nanomaterials is difficult to describe. This is so since these materials are fabricated by various methods, various types of samples are prepared for their examinations, and various methods are

\footnotetext{
*Corresponding author: kitserpes@upatras.gr
} 
employed for measuring their properties.

However, whichever is the production technique, nanocrystalline materials undergo grain coarsening which is a thermodynamic driven process. Due to the monotonic reduction of the Gibbs free energy versus the grain size, nanocrystalline materials exhibit advanced mechanical behaviour, which, however, is prone to thermodynamic instability at elevated temperatures. The most promising production technique is the High Energy Ball Milling in which the grain size decrease with the milling time down to a constant value which varies with the melting temperature of the material. This phenomenon implies a balance between defect creation and recovery during deformation. Provided the problems of contamination during the milling process (by attritors or absence of inert gas environment) and powder consolidation without coarsening are solved, mechanical attrition could be used for producing nanocrystalline materials in notable quantities. Additional problems in the production of nanocrystalline materials are the high energy consumption and the timeconsuming process.

From the above, it becomes evident that experimental characterization of the mechanical behavior of nanocrystalline materials is a very difficult task due to the inability of the research community to produce enough material for test specimens. On the other hand, these technological limitations have been the impetus for the development of models capable of correlating the material structure (grain size, volume fraction of each phase, etc) with the mechanical properties of nanocrystalline materials.

In the present work, a numerical model is developed to predict the Young's modulus and Shear modulus of nanocrystalline materials using a Finite Element Analysis. The model is based on Representative Volume Elements (RVE) in which the microstructure of the material is described using the Voronoi tessellation algorithm and its results are in compliance with several scientific publications.

\section{Analytical approaches}

\subsection{Rule of mixtures}

The following model is presented in its general formulation. The basic idea (Fig. 1) of the model assumes that $\mathrm{NC}$ material is a composite consisting of two distinct phases: the grain core or crystallite (inclusions) and the grain boundaries (matrix). In addition, a perfect bonding between the two phases is assumed. To simplify the analysis, grain boundary triple junctions are incorporated in the grain boundaries in terms of volume. This simplification should not have a major influence on the results for materials with a grain size greater than $10 \mathrm{~nm}$ since the volume.
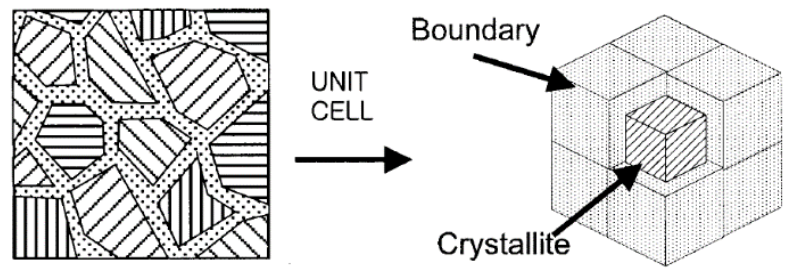

Fig. 1. Illustration of Rule of Mixture approach

Nanocrystalline materials generally refer to the class of materials whose average grain size is below $100 \mathrm{~nm}$. As many atoms reside in the grain boundary regions in this case the volume fraction of the grain-boundary phase is not zero. In terms of the grain size (diameter) $\mathrm{d}$ and grain-boundary thickness $\mathrm{t}$, the volume fraction of the grains can be approximated by 


$$
V F_{c r}=\frac{d^{3}}{(d+t)^{3}}
$$

and that of the grain-boundary phase by $V F_{g b}=1-V F_{c r}$. In addition, the inclusion phase (crystallite) will also be considered homogeneous. In a coarse-grained material we have $t / d \rightarrow 0$; the whole polycrystal is then fully occupied by the grains and its elastic behavior is simply the averaged behavior of these crystallites. But for a nanocrystalline material, for instance at $\mathrm{d}=20 \mathrm{~nm}$ and $\mathrm{t}=1 \mathrm{~nm}$, the volume fraction of the grain-boundary phase is about $14 \%$ and its contribution to the overall elastic behavior presents some fluctuations.

Fig. 1 shows a schematic of the phase mixture model in an NC material. A cubic unit cell of the NC material consists of a crystallite (grain interior) and grain boundaries. It was shown [4] that the results of the finite element calculations for the analysis of plastic deformation in NC materials are almost the same as those obtained using the rule of mixtures. Therefore, in this study, the following simple rule of mixtures based on the volume fractions of the components is used for the analysis of deformation behaviour:

$$
E=V F_{c r} E_{c r}+V F_{g b} E_{g b}
$$

where the subscripts cr and gb refer to crystallite and grain boundary, respectively. The stress in each of the two components of the 'composite' is calculated using the assumption that the strains in both phases are the same and are equal to the macroscopic applied strain.

\subsection{Mean-field homogenization approach (MFH)}

The purpose of mean-field homogenization (MFH) is to compute approximate but accurate estimates of the volume averages of the stress and strain fields, both at the RVE level (macro stresses and strains) and in each phase. It is important to emphasize that MFH does not solve the RVE problem in detail, and therefore does not compute the detailed micro stress and strain fields in each phase.

In the current work, we study simple two-phase composites made of a matrix material reinforced with a number of identical inclusions (I), having all the same material, shape and orientation. We use subscripts 0 for the matrix and 1 for the inclusions phase. The volume fractions in the two phases are such that $V F_{0}+V F_{1}=1$. The volume averages of the strain field over the RVE, the matrix phase and the inclusion phase are related as follows:

$$
\langle\varepsilon\rangle_{\omega}=V F_{0}\langle\varepsilon\rangle_{\omega o}+V F_{1}\langle\varepsilon\rangle_{\omega 1}
$$

Actually, this identity holds for any micro field (e.g., stress field). Any MFH model can be defined by so-called strain concentration tensors such that:

$$
\langle\varepsilon\rangle_{\omega 1}=B^{\varepsilon}:\langle\varepsilon\rangle_{\omega o}, \quad\langle\varepsilon\rangle_{\omega 1}=A^{\varepsilon}:\langle\varepsilon\rangle_{\omega}
$$

The volume average of strain over all inclusions is related to the volume average of strain over the matrix phase via the first tensor, and to the volume average of strain over the entire RVE (macro strain) with the second tensor. The two strain concentration tensors are not independent. Indeed, the second one can be computed from the first one:

$$
A^{\varepsilon}=B^{\varepsilon}:\left[V F_{1} B^{\varepsilon}+\left(1-V F_{1}\right) I\right]^{-1}
$$

These results are valid for any material model for either phase. For any homogenization model defined by a strain concentration tensor, the macro stiffness (sub. 0 for matrix and 1 for inclusions) is 


$$
\bar{C}=\left[V F_{1} C_{1}: B^{\varepsilon}+\left(1-V F_{1}\right) C_{0}\right]:\left[V F_{1} B^{\varepsilon}+\left(1-V F_{1}\right) I\right]^{-1}
$$

\subsubsection{Mori-Tanaka approach}

An infinite solid body is subjected to linear displacements on its boundary corresponding to a uniform remote strain $\mathrm{E}$. The body is made of a matrix phase of uniform stiffness $\mathrm{C}_{0}$ in which is embedded a single ellipsoidal inclusion (I) of uniform stiffness $\mathrm{C}_{1}$ (Fig.2 ).

Using Eshelby's solution, this problem can be solved in closed form. It is found that the strain inside the inclusion (I) is uniform and related to the remote strain as follows:

$$
\varepsilon(x)=H^{\varepsilon}\left(I, C_{0}, C_{1}\right): E
$$

where $H^{\varepsilon}$ is the single inclusion strain concentration tensor, defined as follows:

$$
H^{\varepsilon}\left(I, C_{0}, C_{1}\right)=\left\{I+\zeta\left(I, C_{0}\right): C_{0}^{-1}:\left[C_{1}-C_{0}\right]\right\}^{-1}
$$

Another tensor which plays an important role is Hill's (polarization) tensor defined as:

$$
P^{\varepsilon}\left(I, C_{0}\right)=\zeta\left(I, C_{0}\right): C_{0}^{-1}
$$

The solution of the single inclusion problem is the cornerstone of well-known and successful MFH models.

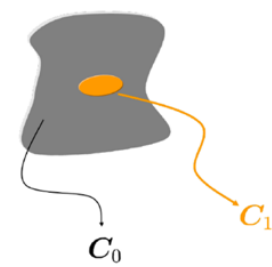

Fig. 2. Single inclusion embedded in an infinite body.

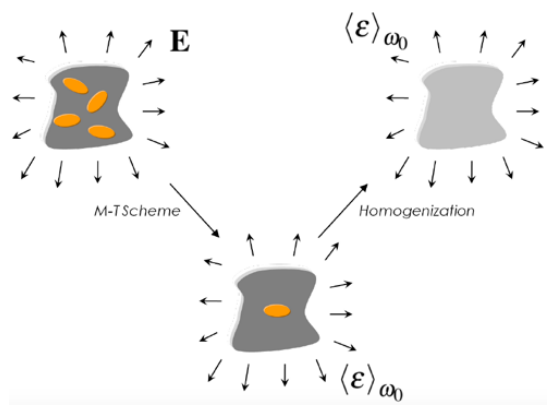

Fig. 3. Illustration of the Mori-Tanaka (M-T) model.

This model was proposed by Mori and Tanaka [5]. The derivation is based on an approximate use of Eshelby's solution. It is found that the strain concentration tensor relating the volume average of strain over all inclusions to the mean matrix strain is given by:

$$
B^{\varepsilon}=H^{\varepsilon}\left(I, C_{0}, C_{1}\right)
$$

which is exactly the strain concentration tensor of the single inclusion problem. This led Benveniste [6] to give the following simple interpretation of the Mori-Tanaka (M-T) model. Each inclusion in the real RVE behaves as if it was isolated in the real matrix. The body is 
infinite and subjected to the average matrix strains in the real RVE as the far field (remote) strain. This is illustrated in above figure 3. The M-T model is very successful in predicting the effective properties of two-phase composites. In theory, it is restricted to moderate volume fractions of inclusions (less than $25 \%$ ) but in practice it can give good predictions well beyond this range.

\section{Numerical approach}

Atomistic simulation methods provide unprecedented insight into the structural behaviour of $\mathrm{NC}$ materials. However, their exploitation for predicting the respective mechanical properties is very difficult due to inherent restrictions of the methods. Therefore, a numerical model of $\mathrm{NC}$ materials aims to provide a tool for the design-by-analysis of the essential NC material microstructural features in order to obtain the desired mechanical behaviour.

The proposed approach is based on the development of Representative Volume Elements (RVE) of the NC material. RVE is the smallest material volume from the simulation of which a macro-structural material property can be estimated. Due to the small size of RVEs (in the case of NC materials they are about $100 \mathrm{~nm}$ edge cubes) detailed morphology of materials microstructures using different types of algorithms can be modeled. Observation of NC materials SEM/TEM [7] images (Fig. 4) from several publications revealed that NC materials consist of randomly polyhedral shaped grains. In order to represent the realistic microstructure of NC materials into RVE, the micro-structure geometry has been developed using Voronoi tessellation algorithm. In each RVE, detailed three-dimensional modelling of the grain and grain boundaries as randomly-shaped sub-volumes is performed (Fig. 5). As considered volume fraction of grains and grain boundaries play a significant role on the overall calculated mechanical behaviour, these volume fractions can be parametrically defined in the NC model.

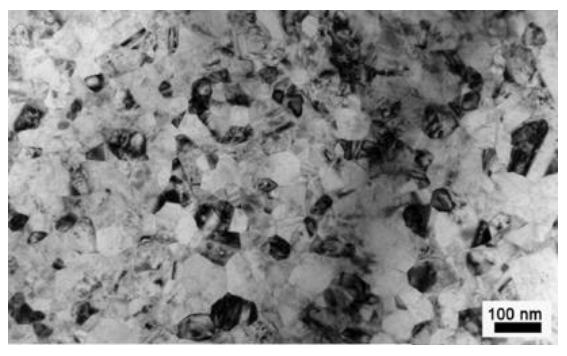

Fig. 4. A TEM bright-field image of the NC Copper [7]

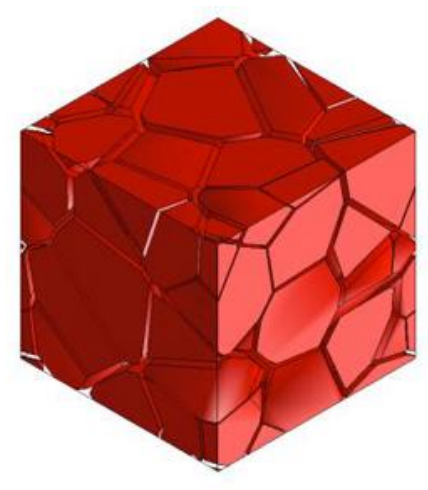

Fig. 5. Unmeshed Voronoi Tesselation

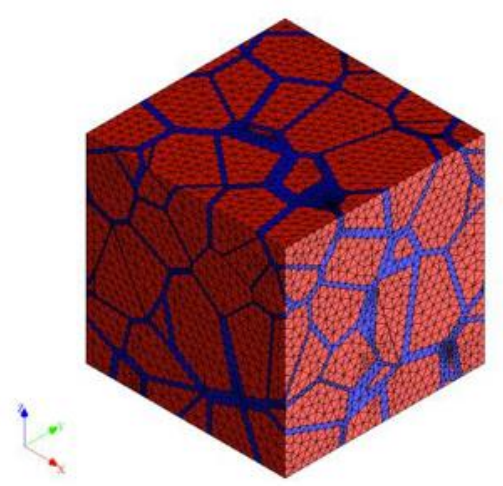

Fig. 6. Representative RVE of a NC material 
The RVE geometrical model is meshed using tetrahedral finite elements (Fig. 6), proper material laws at each sub-volume are assigned and the RVE is loaded under representative loading conditions. The mechanical parameters assumed in the computations are summarized in Table 1 .

Table 1. Mechanical properties assumed in the computations.

\begin{tabular}{|c|c|c|}
\hline & Young modulus [GPa] & Poisson number \\
\hline Grain interiors & 120 & 0.336 \\
\hline Grain boundaries & $0.8 * 120$ & 0.336 \\
\hline
\end{tabular}

The elastic modulus of the grain boundaries, $\mathrm{E}_{\mathrm{gb}}$, was $80 \%$ of the value for the grain interiors, $E_{\mathrm{cr}}$. The assumption of the lower value of the grain boundary elastic constant was based on the results of ab initio computations reported in [8].

The elastic moduli of the material (Young's Modulus of Elasticity and Shear Modulus) can be numerically predicted without the need to perform an extensive mechanical test campaign. For validation purposes, a limited number of experiments is necessary. The developed methodology will provide the means to design the essential $\mathrm{NC}$ material microstructure based on the required material properties.

\section{Results and discussions}

Three-dimensional Voronoi particle RVE models were randomly created using the technique of Christoffersen [9] grain algorithm. Several investigations were made in order to determine the influence of the size of the Voronoi particles (grains) on the effective material properties of these composite-like materials (NC materials) and the influence of grain boundary thickness. The results showed that the influence of the size of the Voronoi particles on the effective material properties was not significant in the linear elastic case. Taking this fact into consideration and, using different sizes of the Voronoi particles, the effective material properties of these composites were obtained for up to $95 \%$ volume fractions.

The results of the numerical approach were compared with different analytical methods which are Mori-Tanaka estimates (M-T) and Rule of Mixtures Method (ROM). Also several investigations were made to determine the effect of the grain boundary thickness on the effective material properties of these materials.
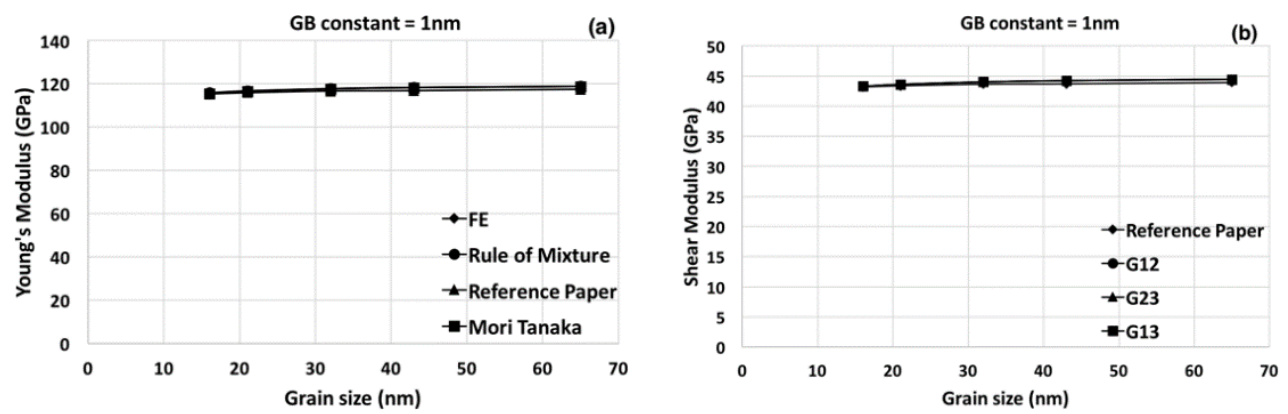

Fig. 7. The numerical and analytical results of nanocrystalline materials for Young's Modulus (a) and Shear Modulus (b) taking the grain boundary thickness as a constant value of $1 \mathrm{~nm}$.

Different studies were made to determine the effect of the size of the Voronoi particles on effective material properties of these NC materials. Fig. 7 explains the effect of the size of the Voronoi particles on the effective material properties. Here the thickness of the grain boundary remains constant and by varying the size of the particles, effective material 
properties were obtained at $83 \%$ - 95\% volume fraction. From Fig.7, it can be observed that there were no significant variations by changing the size of the Voronoi particles on the effective material properties.

Although slight variations can be observed in the effective material properties with change in the size of the particles, these might be due to greater number of particles inside the RVE by reducing its size for the same volume fraction.

Fig 7. shows also the comparison between the effective material properties of the numerical homogenization techniques and Mori-Tanaka approximations for the assumption that grains have been simulated like spherical particles. Furthermore, the results of Rule of Mixtures have been shown accompanied with the aforementioned comparison, but this analytical method does not take into consideration the shape of RVEs' inclusions.
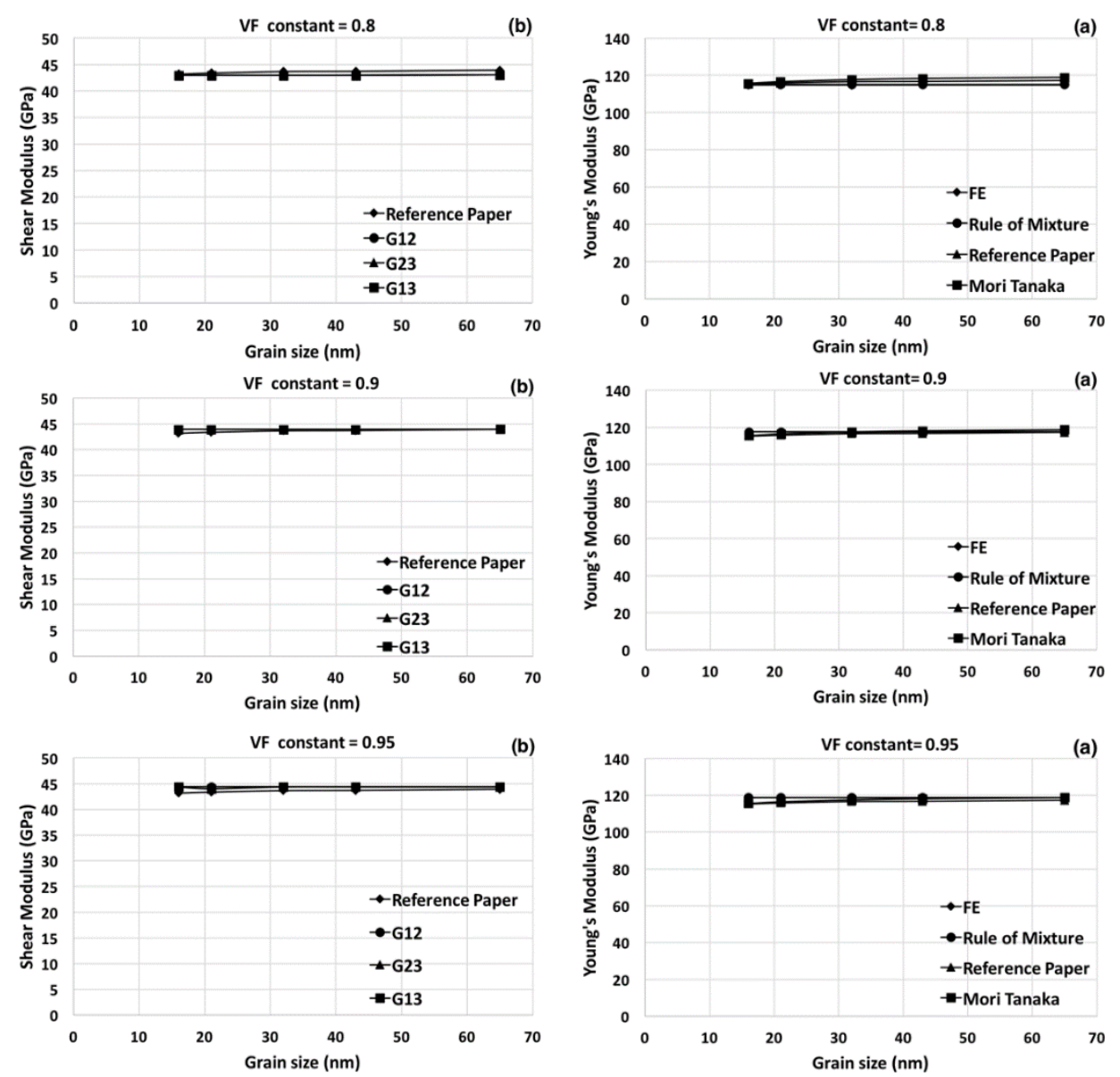

Fig. 8. The numerical and analytical results of NC materials for Young's Modulus (a) and Shear Modulus (b) taking the volume fraction of grains as a constant value of $80 \%, 90 \%$ and $95 \%$ respectively.

From Fig. 8, in which RVEs consist of $80 \%, 90 \%$ and $95 \%$ constant volume fraction of grains respectively, it can be observed that the differences of the numerical effective material properties were very small between the analytical methods. From these studies it can be concluded that the effective material properties of the Voronoi particle reinforced composites (NC materials) depend only on the volume fraction. The size of the particles has not a significant influence on the effective material properties in the linear elastic case. The above 
numerical and analytical results are in absolute compliance with the numerical results of the [10] which are named in the Fig.8 as "Reference Paper".

\section{Conclusions}

The numerical homogenization tool has been developed for the evaluation of the effective material properties of Voronoi particles reinforced materials. The elastic moduli of the Voronoi particle reinforced composites obtained using these tools were compared with the results of different analytical methods. Our numerical predictions were close to the results of Mori-Tanaka and Rule of Mixture approximations. Several investigations were made to determine the influence of the size of the Voronoi particles on the effective material properties. The results showed that the effective material properties depend mainly on the volume fraction. There were no significant variations with respect to the change in size of the particles. This statement is valid for linear elastic case for the evaluation of the effective material properties only. There may be some influence in non-linear case, de-bonding and damage predictions. Further investigations have to be carried out to determine the influence of the size of the Voronoi particles on the elastic-plastic behavior of nanocrystalline materials at macro-level. A generalized procedure has been developed to calculate different effective coefficients for all desired volume fractions based on the Digimat Software ( ). This tool reduces the workload and time and can be used as a template to evaluate the effective coefficients of randomly distributed Voronoi particle reinforced composites.

The ICARUS project has received funding from the European Union's Horizon 2020 research and innovation programme under grant agreement No 713514 .

\section{References}

1. K. Kumar, H. Van Swygenhoven, S. Suresh, Acta Mater. 51, 19 (2003).

2. M. Meyers, A. Mishra, D. Benson, Prog. Mater. Sci. 51, 4 (2006).

3. M. Kulczyk, W. Pachla, A. Mazur., R. Diduszko, H. Garbacz, M. Lewandowska, W. Łojkowski, K. Kurzydłowski, Mater. Sci., 23, 3 (2005)

4. H. Kim, C. Suryanarayana, S. Kim, B. Chun., Powder Metall. 41, 3 (1998).

5. T. Mori, K. Tanaka, Acta Metall. 21, 5 (1973).

6. Y. Benveniste, Mech. Mater. 6, 2 (1987).

7. J. Hu, S. Han, G. Sun, S. Sun, Z. Jiang, G. Wang, J. Lian, Mater. Sci. Eng., A 618, pp.621628 (2014)

8. M. Muzyk, PhD Thesis, Warsaw University of Technology (2010).

9. J. Christoffersen, J. Mech. Phy. Solids 31, 1 (1983).

10. R. Dobosz, M. Lewandowska, K. Kurzydlowski, Scr. Mater. 61, 4 (2012). 